\title{
Application of AHP and Fuzzy MADM to Evaluation of Bus System Performance in Taipei City
}

\author{
Gwo-Hshiung Tzeng and Rong-Tsu Wang \\ Energy Reseach Group,and Institute of Traffic and Transportation \\ National Chiao Tung University 114-4F,Sec.1,Chung Hsiao W.Rd.,Taipei100,Taiwan,R.O.C.
}

\begin{abstract}
In recently, urban traffic became more worse in Taipei city. The solution approach has to depend on universalization of public transportation. However, we need first a perfomance method for evaluating transportation systems. This paper focus on establishing a complete, objective and easier evaluating process. The selection of performance evaluation indices, the weight of indices by using AHP method and the priority rankings of all bus systems according to the concept of fuzzy MADM are included to describe.

In this paper the hierarchical structure of bus performance evaluation indices was constructed by considering three aspects of supply side(bus firms), demand side(passengers) and supervise side(policy): then sixteen evaluation indices were selected in this bierarchical structure. The weights of all evaluation indices was obtained by using the hierarchy concepts of AHP and its eigenvector from decision-groups of bus operators,passengers, government autborities and scholar experts. Then the priority rankings of all bus systems-TOPSIS were ranked by applying the outranking of fuzzy MADM.

Finally, ten bus firms in Taipei city were illustrated and ranked by using this evaluation process. It is show that simplify the complexity of fuzzy application to the bus system evaluation. The results also be more suited to the characteristics of this problems.
\end{abstract}

Keywords: Bus system performance, performance evaluation indices, analytic hierarchy process(AHP), fuzzy multiple attribute decision making.

\section{Introduction}

In recent years, air pollution that is accompanied by increasing urban traffic congestion has only gone for the worse. And the solution to the problem depends very much on the prevalent public transportation, while public bus plays a rather significant role in it. Of course, it is a matter of the highest urgency as how the performance of public bus system can be increased so as to attract more passenger. Initially, how can the performance index selecting and evaluating public system be derived? The task can be conducted by evaluating the managment and operation objectives of the public-owned enterprises - their efficiency and performance (Tally, and Anderson, 1981; Fielding and Anderson, 1984; Field et al., 1985a; Tantoriboon et al., 1993). Different manners of evaluation and selection have been conducted, and some have been evaluated and selected from the hardware equipment and passenger service standard (Transportation Department of the Taipei Municipal Administration, 1991); some have been evaluated and selected from the five major objectives in pursuit by the public bus system (Chang and Tsai, 1993); some have been evaluated and selected first by separating bus into peer groups, then the performance of various transportation systems in the same peer group is compared (Fielding et al., 1985b); others would first break up transportation zones into different services areas, next the performance of the transportation system within the area is evaluated and selected (Lan , 1983). This study considers that transportation industry is within the domain of regulation economy, and bias is most likely to be brought forth should the enterprise be viewed from any particular perspective. lacking in its panorama. Therefore, three individual perspectives as the supply aspect of bus operator, the demand aspect of passenger, and the aspect of supervision policy being the most important and special part of regulation economy are employed as foundation for selecting performance index.

Though a wide selection of literatures have been done evaluating the performance of transportation system, only countable few have worked on the weight size of performance index and these" few were, unfortunately, conducted from unrefined and average expert viewpoint, which merely renders the derived weight size being subjective and unreasonable. Thus, this study will employ hierarchy concept and eigenvector method of analytic hierarchy process (AHP) to analyze and obtain the weight size of each of the performance indices. 
Then in the process evaluating the performance of transportation system, there are always such fuzzy expressions as "high", "medium", and "low" seen in the measurement of qualitative indices, so the generally binary logic concept is inapplicable in this situation. This study utilizes the administration and alternative ranking methods endowed in the fuzzy. expressions of fuzzy multiple attribute decision making (FMCDM), then takes operators of bus crmpanies as altematives and evaluation index as attribute or criterion, and the ranking among bus system is performed among each of the derived index weights. As for the method of ranking, this study suggested using TOPSIS to handle the issue as the two premises of TOPSIS - known attribute weight and monotonically increasing or monotonically decreasing features - conform to the characteristics of performance evaluation problem of public bus system. At the end, this paper will illustrate with examples with the ten bus companies in Taipei, and employ such evaluation procedures to conduct the task of prionity ranking anong these systems.

\section{Sejection of Performance Evaluation Index of} the Bus System

In the past, there are various manners choosing perfomance evaluation indices, such as the one by. Fielding et al. (1978), which put forward the strucnure of performance concept model: cost efficiency, cost effectiveness, and service effectiveness as bases for selection; the transportation department of Taipei municipal administration (1993) separated performance indices into four categories as vehicle and station, quantified passenger service standard, qualitative passenger service standard, and other major policy that goes hand in band with transportation department; Chang Yu- Hem (1993) exploited five primary objectives as safety, comfor, economy, convenience, and social responsibility for his selection.Using these operator-sided, passenger-sided, or the more idealistic transportation objective to select performance evaluation indices could ultinately render results that would make passengers still displeased after improvement if the measures employed are operator-sided, or the operators are being displeased and lowered of their willingness to improve as the suggested measures are passenger-sided.

This study believes that transportation industry is within the domain of regulation economy, and it is better that the view of economics be taken so as to provide a more objective and unbiased perspective. So, the supply aspect of economics is selected to evaluate the managment and operation performance of the operators, the demand aspect to satisfy the service standard, and the inevitable supervision aspect - the fitness of policy -- of regulation economics as a whole to cope with the consideration of the three perspectives. The evaluation structure is as indicated in Figure ?

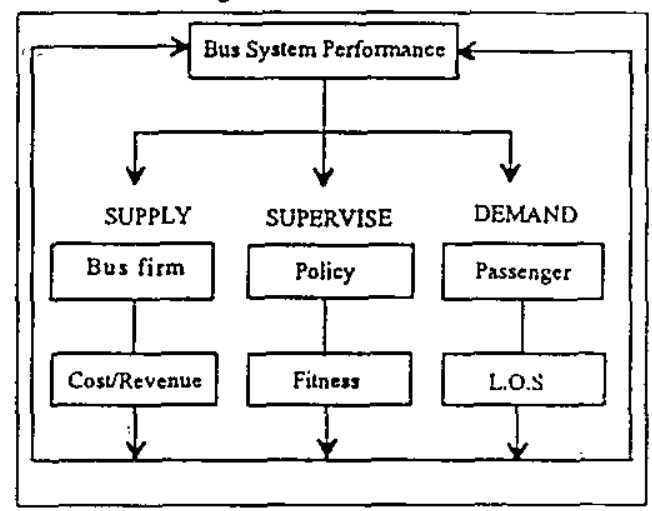

Figure 1. The Performance Evaluation Model of Bus System

For the evaluation of supply aspect, it is mainly that the viewpoint of operator is taken into account for selecting the performance evaluation index, and the project done by Allen et al. (1976) and Fielding et al. (1985a) are based upon as references. As a matter of fact, what concerns operators is nothing but four components -- employee, vehicle, operation situation, and the final net income. In view of it, this study will present four supply aspects of the evaluation indices, which are indicated in Table 1

As for the evaluation of demand aspect, it is primarily that the viewpoint of passenger is taken into deliberation for selecting performance evaluation index, while the service standard encountered by passenger is of the most representative. Various relevant studies have already been done on service standard, such as in Bakker (1976) which exploited In-vehicle congestion as its evaluation index; Alter (1976) utilized availability, traveling time, frequency reliability, directness of service, bus frequency, and passenger density to measure; Allen et al. (1976) divided the characteristics of transportation systems into quantifiable indices as frequency, capacity, and degree of road congestion, and into qualitative indices as driving speed of bus, feasibility, comfor. convenience, safety, special service, pollution, and bus fee aspects to describe the system performance and service standard. This study, based upon the literature review as well as on the availability and measurability of data, will put forward eight evaluation indices of the demand aspect, and the evaluation indices are as shown in Table 2. 
Table 1. The Establishment of Performance Evaluation Indicies of the Supply Aspect

\begin{tabular}{|l|l|l|}
\hline Quardrant & Performance Index & \multicolumn{1}{|c|}{ Measurement } \\
\hline \multirow{4}{*}{$\begin{array}{l}\text { Supply } \\
\text { Side }\end{array}$} & Staff & Total Revenue/Total staff number \\
\cline { 2 - 3 } & Vehicle & Total Revenue/Total vehicle-km \\
\cline { 2 - 3 } & Maintenace & Total vehicle-km/Total maintenace cost \\
\cline { 2 - 3 } & Revenue/cost & Total revenue/Total cost \\
\hline
\end{tabular}

For the evaluation of supervision, it is mainly that the viewpoint of government is taken into thought for selecting performance index. Since the government plays supervisory role, the performance evaluation of bus system will be based upon the fitness of relevant policy to the system. As a result, the consideration of supervision executed in this stady is based on the policy fitness for its measurement, however, due to the timing constraint of policy fomulation and implementation it is, therefore, those important policies highly regarded by
After Table 1, Table 2, and Table 3 have been integrated, this study has altogether selected sixteen performance evaluation indices, and ten are quantitative indices and six are qualitative indices. Quantitative indices can be derived from objectuve information, while qualitative indices must be obtained through questionnaire investigation. Except those qualitative indices of supervision aspect such as the information on management indices of bus-stop and station that must be obtained from the routine

Table 2. The Establishment of Performance Evaluation lndices of the Demand Aspect

\begin{tabular}{|c|c|c|c|}
\hline Quadrant. & Performance & Index & Measurement \\
\hline \multirow{8}{*}{$\begin{array}{l}\text { Demand } \\
\text { Side }\end{array}$} & & Accident rate & Accident on duty/Total operation mileage \\
\hline & Safty & $\begin{array}{c}\text { Average } \\
\text { vehicle age }\end{array}$ & Evehicle number $x i$ of age $i$ year/total vehicle number \\
\hline & \multirow{3}{*}{ Comfort } & $\begin{array}{l}\text { In-vehicle } \\
\text { congestion }\end{array}$ & $\star$ \\
\hline & & $\begin{array}{l}\text { proportion of } \\
\text { air condition } \\
\text { vehicle }\end{array}$ & Number of air condition vehicle/Total operation vehicle \\
\hline & & $\begin{array}{l}\text { Driving } \\
\text { Stable }\end{array}$ & $\star$ \\
\hline & \multirow[t]{2}{*}{ Convenience } & $\begin{array}{l}\text { Frequence } \\
\text { Reliability }\end{array}$ & $\star$ \\
\hline & & $\begin{array}{l}\text { Chang bus } \\
\text { Convenience }\end{array}$ & $\star$ \\
\hline & \multicolumn{2}{|c|}{ Service Attitude } & $\star$ \\
\hline
\end{tabular}

Remark: Asterisk indicates that such index is of qualitative index, enshrined with fuzzy existence, and the method of managment will be elaborated in the fourth part.

the transportation department of Taipei municipal administration that are founded on for selecting performance indices. Four indices have been selected and are as shown in Table 3. supervision results of transportation department, others can only be obtained from the questionnare investigation of customer satisfaction.

III. The Establishment of Performance Evaluation Index Weight 
Generally speaking, once when the performance evaluation indices have been selected, the weights of these indices are mostly taken as consistent. So, when Table 3 The Establishment of Performance Evaluation Indices of the Supervision Aspect

established, which is as shown in Figure 2.
ce Evaluation Indices of the Supervision Aspect

\begin{tabular}{|c|c|c|}
\hline Quadrant & Performance Index & Measurement \\
\hline & Environment Pollution & $\begin{array}{c}\text { Punishment number on pollution (fiscal } \\
\text { year)/Total vehical number }\end{array}$ \\
\hline $\begin{array}{c}\text { Supervision } \\
\text { Side }\end{array}$ & Management of Driving Safe & $\begin{array}{c}\text { Accident number on duty(fiscal year)/The } \\
\text { number inform municipal administration }\end{array}$ \\
\cline { 2 - 3 } & Management of Bus-stop Station & $\begin{array}{c}\text { Advertisement number on bus of each bus } \\
\text { co/The number apply to municipal } \\
\text { administration }\end{array}$ \\
\cline { 2 - 3 } & Advertisement on bus index & \\
\hline
\end{tabular}

bus company operators with inferior system performance try to improve, the order is to begin from the index that performs the worst, rather than from the indices that either the operator or passenger urgently desires for improvement, because if such being the case the efforts will only be halved with incommensurable outcome. Also, the system performance derived will be of average level rather than of more meaningful weighted average level, and that will render the evaluation result meaningless. In view of the case, we are going to investigate the establishment method of performance evaluation index weight.

There are methods to establish weight (Hwang and Lin, 1987), such as eigenvector method, weighted minimal square method, entropy method and so on, Nevertheless, the selection of methods depends on the nature of problem, since the performance evaluation of bus system is both complicated and involve extensive aspects of problems it requires such a method that can consider from multiple hierarchies as well as systemize problems. In such a way, the characteristics of all problems can then be comprehensively embraced by it. Therefore, this study has selected AHP as the basis to establish weight, and the method was put forward by Saaty in 1971. It is hoped that complex problem can be systemized into various areas for hierarchical analysis so as to be devised of hierarchical structure (Hwang and Lin, 1981; Saaty, 1980; Sugiyama, 1981), furthermore, 1-9 rate scale will be executed to the comparative weights among each of the evaluation criteria for pairwise comparison. After then, comparative matrix can be established, and eigenvector as well as eigenvalue can be calculated. At the end, once the greatest eigenvector has been verified of its consistency, the size of relative weight arnong each of the evaluation criteria then be obtained.

Based on the hierarchical nature of AHP, this study has founded itself on such feature to establish weight. Initially, the hierarchical structure of the performance evaluation of bus system is to bc

In Figure 2, the pairwise matrixes among these three objectives are to be contrived by operators of the bus companies, scholars, and experts; of the four indices of the supply aspect, their pairwise comparison matrixes are to be determined by operators of the bus companies; the four indices of the

supervision aspect and the eight indices of the demand aspect are to be jointly contrived of their pairwise matrixes by scholars and experts, authorities in charge of, and consumer group. Then, they are respectively calculated of their comparative weights, and the details of the procedures are as following:

1. To establish hierarchical structure, and the results are as shown in Figure 2.

2. To establish painwise comparison matrix. The elements of a certain hierarchy would base on a certain element of the preceding hierarchy as its evaluation criterion so as to conduct its pairvise comparison among its elements. For instance, take cost/benefit as element, and conduct such painwise comparison among staff performance, vehicle performance, maintenance performance and benefit/cost ratio. And its elements of pairwise matrixes are as indicated:

$(A)=\left[\begin{array}{cccc}\text { Staff } & \text { Veh } & \text { Maintenace } & \mathrm{B} / \mathrm{C} \\ 1 & a_{12} & a_{13} & a_{14} \\ 1 / a_{12} & 1 & a_{23} & a_{24} \\ 1 / a_{13} & 1 / a_{23} & 1 & a_{34} \\ 1 / a_{14} & 1 / a_{24} & 1 / a_{34} & 1\end{array}\right]$

For same reason, the pairwise comparison marrixes of fitness and service standard can be respectively established. 


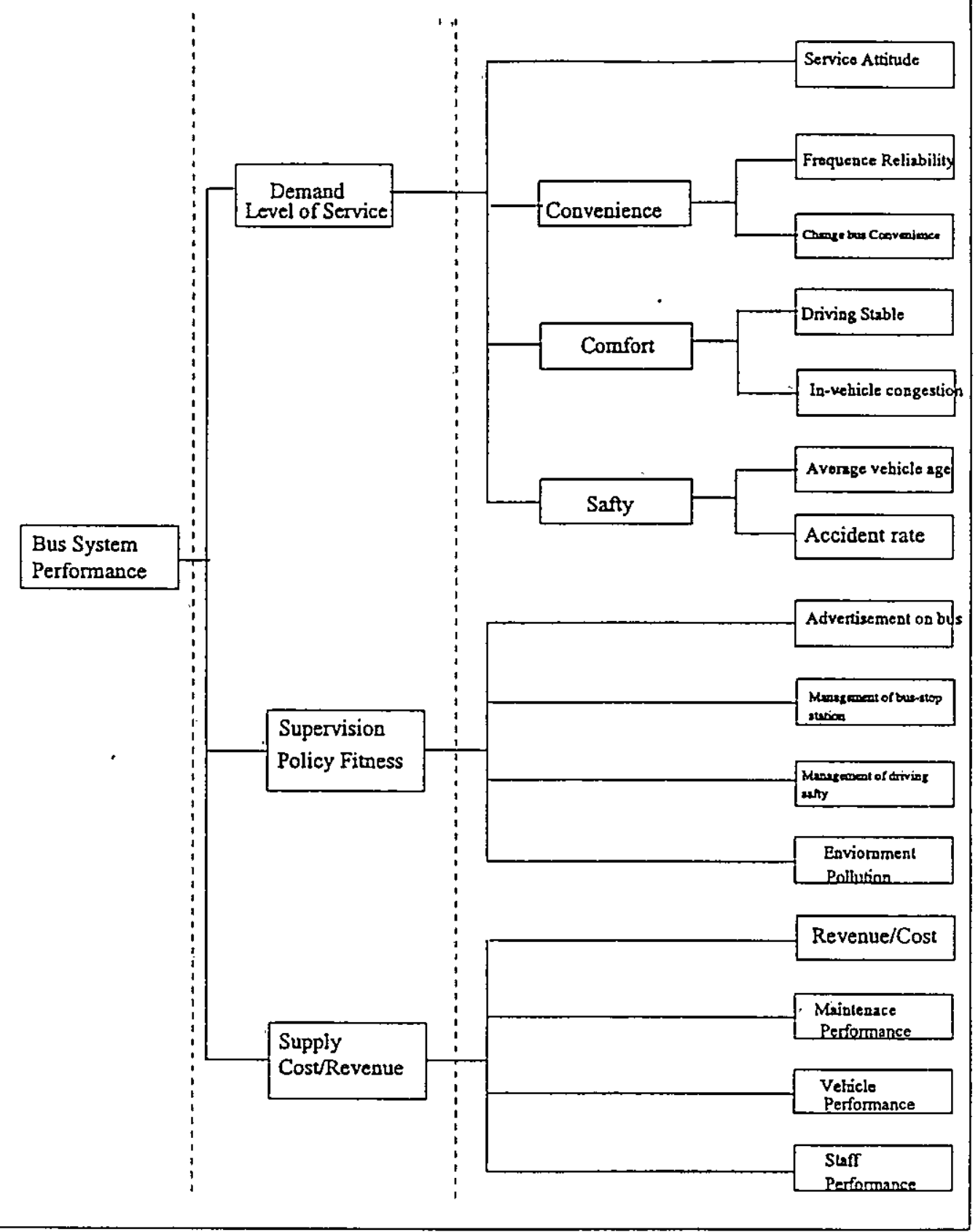

Figure 2. The Hierarchical Structure of the Performance Evaluation of Bus System

3. Calculation of eigenvalue and eigenvector. Use the solution-finding method for eigenvalue seen often in the numerical valute analysis to socate either eigenvector or priority vector. If $\lambda$ be the eigenvalue for pairwise comparison matrix, then,

$$
\begin{gathered}
A \cdot X=\lambda \cdot X \\
(A-\lambda D) \cdot X=0
\end{gathered}
$$

$X$ is the eigenvector of $A$.

4. Verification of consistency and the establishment of relative weight among performonce indices. This study has started from the three objectives to conduct their pairwiso comparison, and has obtained their relative weights. Then, the pairwise comparisons are performed among the performance evaluation indices of the supply aspect, demand aspect, and supervision aspect, so their relative weights are subsequently derived. Moreover, due to the fact that there are as many as eight evaluation indices occurred in regard to the demand aspect and it is hoped that the inconvenience of filling-in can be avoided, therefore, 
the relative weights are to be first derived from the four integral indices as safety, comfort, convenience and service standard before pairwise comparative matrixes are contrived. Afterwards, the pairwise comparisons under each of the evaluation indices as safety, comfor, convenience and service standard are administered, as a result of it, the study result can become more representative as of the higher accuracy obtained than fllling-in.

However, consistency verification has to be performed before the derived weight is being utilized. The so-called consistency verification is to measure the degree of reasonableness in regard to the judgment done during the evaluation process. Saaty suggested that the value of consistency index (C.I.) should be around 0.1 , and the consistency ratio (C.R.) should be smaller than 0.1 .

$$
\begin{aligned}
& C . I .=\left(\lambda_{\max }-r\right) /(r-1) \\
& C . R .=C . I . / R . I .
\end{aligned}
$$

$\lambda_{\text {max. }}$ is the largest eigenvalue; $m$ indicates the number of operators of bus companies that await to be evaluated; R.I. is the C.I. value occurred under different orders of pairwise comparison matrixes, and it is called random index (R.I.). Its values are as shown in Table 4.

Table 4. Random index Table

\begin{tabular}{|l|r|r|r|r|r|r|r|}
\hline $\begin{array}{l}\text { Level } \\
\text { number }\end{array}$ & 1 & 2 & 3 & 4 & 5 & 6 & 7 \\
\hline R.I. & 0 & 0 & 0.58 & 0.9 & 1.12 & 1.24 & 1.32 \\
\hline
\end{tabular}

\begin{tabular}{|l|r|r|r|r|r|r|r|r|}
\hline $\begin{array}{l}\text { Level } \\
\text { number }\end{array}$ & 8 & 9 & 10 & 11 & 12 & 13 & 14 & 15 \\
\hline R.I. & 1.41 & 1.45 & 1.49 & 1.51 & 1.48 & 1.56 & 1.57 & 1.58 \\
\hline
\end{tabular}

Once the consistency verification has been through, the weights of each of the performance indices can be obtained after the eigenvector $X$ is standardized, $\gamma=$ $\left(\gamma_{1}, \tau_{2}, \ldots, \gamma_{n}\right)^{t}$ and is as shown in Table 5 afier classification.

Table 5 The Weight of Performance Evaluation

\begin{tabular}{|c|c|c|c|}
\hline $\begin{array}{l}\text { Pettomusice } \\
\text { Itadex }\end{array}$ & weight & $\begin{array}{l}\text { Pertornuntes } \\
\text { Index }\end{array}$ & wesigs! \\
\hline Sum & $a_{i}, \gamma_{1}$ & \begin{tabular}{|l} 
Divings \\
Stablece
\end{tabular} & $a_{2}^{*} \beta_{3}^{*}, \gamma_{2}$ \\
\hline Venoles & $\alpha, \gamma_{1}$, & $\begin{array}{l}\text { Canges bus } \\
\text { rooventemes }\end{array}$ & $a_{2}-\beta_{1}, \tau_{4}$ \\
\hline Mannienace & $a_{1}, \gamma_{1}$ & $\left\{\begin{array}{l}\text { Frequence } \\
\text { rellabsuluy }\end{array}\right.$ & $a_{2}-B_{1}{ }_{2} \tau$. \\
\hline sevenuecoses & $\alpha_{1}^{*}, \gamma$ & $\begin{array}{l}\text { Sorvice } \\
\text { santroges }\end{array}$ & $\beta$ \\
\hline Accsdent rale & $\alpha_{2} \cdot \beta_{1} \cdot \gamma_{2}$ & $\begin{array}{l}\text { Envugonment } \\
\text { polluston }\end{array}$ & $a, \cdots, r$ \\
\hline
\end{tabular}
Indices

\begin{tabular}{|c|c|c|c|}
\hline 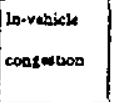 & $\alpha_{2} * \beta_{1}{ }_{2} \gamma_{2}$ & 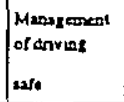 & $\alpha,{ }_{2}^{*} \gamma$ \\
\hline $\begin{array}{l}\text { Averases" } \\
\text { vesticle ase. }\end{array}$ & $a_{2}-\beta_{2}^{*},{ }_{1} \gamma$ & $\begin{array}{l}\text { Manszanent } \\
\text { of trustiop } \\
\text { terton }\end{array}$ & $a,{ }^{*}, \gamma$, \\
\hline $\begin{array}{l}\text { properteon of } \\
\text { ait coodition } \\
\text { velustio }\end{array}$ & $a_{2}^{*} \beta_{2}^{*}{ }_{2} \tau_{3}$ & $\begin{array}{l}\text { Advertisenen } \\
1 \text { os beses }\end{array}$ & $a ;, 7$, \\
\hline
\end{tabular}

Remark 1:the weight values obtained from the pairwise comparison matrixes contrived for demand. supply, and supervision aspects are respectively as $a_{1}, a_{2}, a_{2}$.

Remark 2: the weight values obtained from the painwise comparison matrixes contrived for elements of safery, coziness, convenience, and service attitude. Remark 3: , $r$, indicates the weight value of $p$ criteria obtained from $q$ comparison matrixes.

IV. The Application of Fuzzy Multiple Attribute Decision Making (FMADM)

Once the selection of evaluation index and the establishment of index weight have been completed, the final stage -- bus system performance evaluation -- can be conducted. In the past, population is presumed to be of normal distribution pertaining to the performance evaluation of bus system, and its results can then be ensinrined with comparatively subjective yardstick. Chang Yau Hong and Tsai Chin Tung (1993) introduced fuzzy theory into the performance evaluation of operation service of bus system, and they respectively established the membership function for quantified indices as weli ids qualitative indices. Then, fuzzy synthetic decision and fuzzy multicriteria decision are employed to conduct the evaluation. However, such method when applied oftentimes requires massive amount of and complex computation, and even if it is of crisp set (such as quantified index) it is has to be rendered fuzzy beforehand (to establish membership function). As a matter of fact, administration in such manner does not conform to the design of fuzzy theory - 10 reduce human subjective judgment, and it only enhances interruption during calculation. Thus, as this study is to proceed to the performance evaluation of the system, it will merely establish membership function for qualitative index.

In traditional multiple attribute decision making (MCDM), both attribute (evaluation criterion) weight and performance value are of crisp sets, however, when fuzzy expressions (such as "high", medium", "low") are encountered traditional method would be rendered obsolete. Moreover, the performance evaluation problem of bus system happens to be enshrined with fuzziness, and so this study has brought in fuzzy multiple attribute decision making (FMADM) to evaluate the perfornance of bus system. Though FMADM embraces a wide selection 
of methods (Chen and Hwang, 1992), some are handicapped by two defects. First, its calculation is too complex and the number of alternative that can really work will not exceed ten. Second, even crisp data have to be rendered fuzzy in advance, as a result, it only adds more subjective flavor to the alternatives. With such a picture in mind, this study has cited a new approach forwarded by Chen and Hwang, in addition to each of the index weights obtained in the last section, to conduct the priority ranking of performance of bus system.

The primary characteristic of the concept is to focus on clarifying (being unfuzzed) those fuzzy sets of the materials, then traditional MADM, being aided by hose crisp sets of the original decision making matrix, is utilized to conduct ranking and the aftermath is of simpler calculation as well as of more intelligible concept. Details of the procedures are as following:

First: to render fuzzy data into fuzzy number. During the process of transformation, eight types of scales have been employed in accordance to the difference of expression levels, which are as indicated from part 1 to part VIII of the Figure 3. Take "frequency reliability index" as an instance, should only "high", "medium", and "low" three levels be resorted, then scale II (part II of figure three) can be employed to display its membership degree. The selection of scale is handled as indicated in Table 6, and the selection principle is, at best, to select the smallest scale from the satisfaction expression levels.

Table 6 The Integrated Table of Level Utilization and Scale of Selection

\begin{tabular}{|c|c|c|c|c|c|c|c|c|}
\hline & & & & & & & & \\
\hline Scale & 1 & 2 & 3 & 4 & 5 & 6 & 7 & 8 \\
\hline Expression level & $=$ & $\equiv$ & 五 & 五 & 六 & $t$ & 九 & + \\
\hline None & & & & & & & & $v$ \\
\hline Very low & & & $\checkmark$ & & $\checkmark$ & $\checkmark$ & $\checkmark$ & $\checkmark$ \\
\hline Low-very low & & & & & & & $\checkmark$ & \\
\hline Low & & $\checkmark$ & $v$ & $\vee$ & $\checkmark$ & $\checkmark$ & $\vee$ & $v$ \\
\hline Medium low & & & & $\vee$ & $\vee$ & & $\checkmark$ & $v$ \\
\hline Litlle low & & & & & & $v$ & & $v$ \\
\hline Medium & $\checkmark$ & $\checkmark$ & $\checkmark$ & $\checkmark$ & & $\checkmark$ & $\checkmark$ & $\checkmark$ \\
\hline Little high & & & & & & $v$ & & $\checkmark$ \\
\hline Medium high & & & & $\checkmark$ & $\checkmark$ & & $\checkmark$ & $\checkmark$ \\
\hline High & $\checkmark$ & $v$ & $\checkmark$ & $\checkmark$ & $\checkmark$ & $\checkmark$ & $\checkmark$ & $v$ \\
\hline High-very high & & & & & & & $\checkmark$ & \\
\hline very high & & & $v$ & & $v$ & $v$ & $\checkmark$ & v \\
\hline
\end{tabular}

\begin{tabular}{|l|l|l|l|l|l|l|l|l|}
\hline Excellence & & & & & & & & $\vee$ \\
\hline
\end{tabular}

Second: to render fuzzy number into crisp score. Use the membership score $\mu_{r}(\mathrm{M})$ at the right of the fuzzy number $M$ and the membersipip score $\mu$, (M) at the left of the fuzzy number $M$ to calculate its total membership score $\mu,(M)$, which are as indicated in the following:

$\mu_{r}(M)=\sup _{x}\left[\mu_{m}(x) \cap \mu_{m 2 x}(x)\right]$

$\mu_{l}(M)=\sup _{x}\left[\mu_{\min }(x) \cap \mu_{m}(x)\right]$

$\Rightarrow \mu_{1}=\left[\mu_{r}(M)+1-\mu_{l}(M)\right] / 2$

$\mu_{m}(x)$ :

$\mu_{\max }(x)=\left\{\begin{array}{l}x, 0 \leq x \leq 1 \\ 0,0 \text { thenwise }\end{array}\right.$

$\mu_{\min }(x)=\left\{\begin{array}{l}0, \text { othenvise } \\ 0, x, 0 \leq x \leq 1\end{array}\right.$

The practice is as shown in Figure 4.

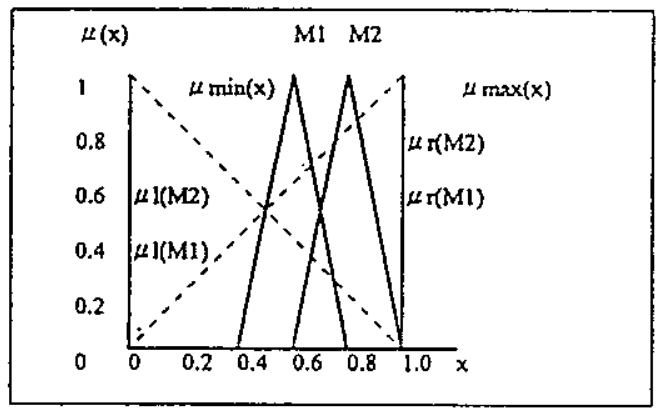

Figure 4.Calculation Chart of the Membership Score

Once the total membership score of each of the calculated fuzzy numerals $M_{1}$ is evened out after being weighted, the exact score (performance value) can be achieved. As the following:

$V_{m j}=\frac{\sum n_{n_{j} \times x_{1}}\left(M_{i}\right)}{N}$

$\mathrm{V}_{\mathrm{mi}}$ : indicates the performance value of $\mathrm{m}$ bus company towards $j$ qualitative index.

$\mathrm{N}$ : indicates the number of the interviewee.

$n_{\text {aij }}$ : considers that the $\mathrm{j}$ qualitative index of $\mathrm{m}$ bus company is of the $M_{i}$ level number of prsons.

$\sum n_{m j i}=N$

$\mu_{i}\left(M_{i}\right)$ : indicates the total membership score of $\mathrm{M}_{i}$ level

Take "frequency reliability" as instance, should its expressions and levels be "high", "medium", and "low" and scale II is employed, then the calculation of the membership score would be as following;

$\mu_{r}($ high $)=1.0$

$\mu_{i}(h i g h)=2 / 3$

$\mu_{t}($ high $)=(1+1-2 / 3) / 2=2 / 3$ 
For same reason, $\mu_{t}($ medium $)=8 / 13, \mu_{t}($ low $)=1 / 6$. In regard to the frequency reliability index of $m$ bus company, there are respectively $n_{1}, n_{2}, n$ number of

persons found in correspondence to responses as "high", "medium", and "low", and its performance value is,

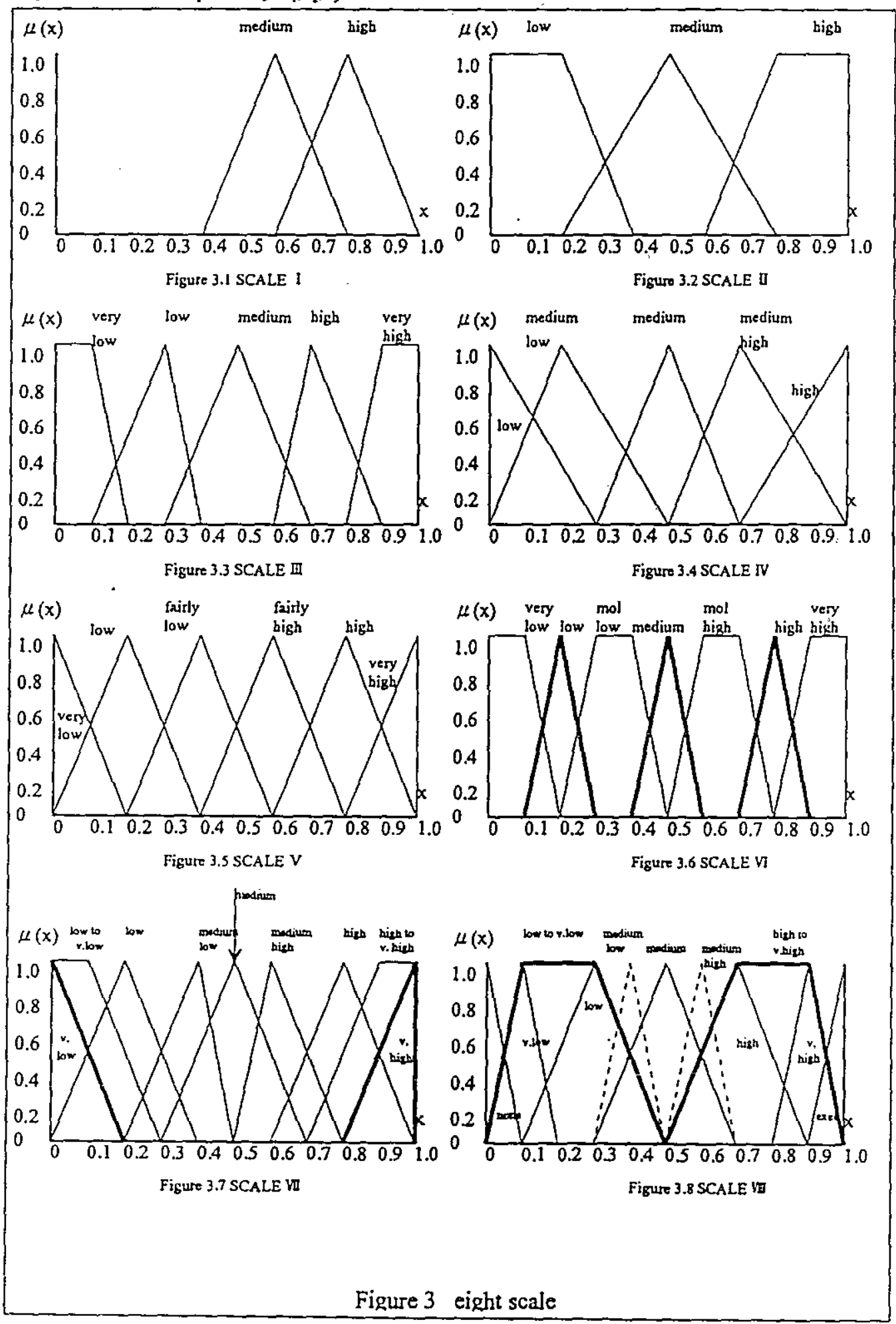




$$
V_{m}=\frac{2 \beta \dot{x}_{1}+8 / 13 \times n_{2}+1 / 6 \times n_{3}}{n_{1}+n_{2}+n_{3}}
$$

Third: any traditional MADM method can be employed to conduct the evaluation ranking pertaining to system performance. Till this stage, all of the performance values in the decision making matrixes are clarified and ranking can be performed by any MADM method, such as TOPSIS, PROMETHEE, ELECTRE, and so forth. This study has employed TOPSIS to administer and the details of which are to be elaborated in the fifth part.

V. Illustration by example - illustrated with the bus system of Taipei City

This study takes the ten bus companies of Taipei City (municipal bus company, Ta Yo, Hsin Hsin, Taipei, San Chung, Metropolitan, Kwong Hwa, Ta Nan, Chi Nan, and Chung Hsin) as its objects of evaluation, while AHP and FMADM are being utilized to evaluate the performance of bus system. At the end, ranking is done by TOPSIS method. This part is divided into three sections: first, AHP is exploited to obtain the relative weights among each of the hierarchies of performance evaluation of the bus system; then the performance value of each of the evaluation indices is to be calculated, including the crisp quantified indices and the qualitative indices derived from using the membership concept of FMADM; at last, TOPSIS of traditional MCDM methods is being utilized to conduct ranking, analyze and review the results derived pertaining to the ten bus companies.

(I) The calculation of each of the evaluation index weights:

With the utilization of AHP, the relative weights are to be figured out among the three objectives (operators' cost/benefit - supply aspect, policy fitness of the authorities in charge - supervision aspect, service standard to passenger -- demand aspect) as the operator of bus company, scholar and expert, authorities in charge of transportation affairs and consumer group, then the relative weights among these three objectives are acquired as shown in Table 7 after the computation procedures is elucidated in the third part.

Table 7 The Relative Weight Values Among Objectives

\begin{tabular}{|l|r|r|l|}
\hline Objective & Supply Side & $\begin{array}{l}\text { Supervision } \\
\text { Side }\end{array}$ & $\begin{array}{l}\text { Demand } \\
\text { Side }\end{array}$ \\
\hline Weight & 0.38 & 0.19 & 0.44 \\
\hline
\end{tabular}

Aftenwards, it is the operators of bus companies who are to contrive the 'weight value of peiformance evaluation index of the supply aspect ; the weight values of performance evaluation indices of the demand and supervision aspects are to be jointly determined by scholar and expert, consumer group, and authorities in charge of transportation affairs And the weight values of performance evaluation indices of the three hierarchies are respectively indicated in Table 8, Table 9, and Table 10.

Table 8 The Relative Weight Values Among the Evaluation Indices of Supply Aspect

\begin{tabular}{|l|r|r|r|r|}
\hline $\begin{array}{l}\text { Evaluation } \\
\text { Index }\end{array}$ & Suff & Vehicle & Maintenace & Revenue/Cost \\
\hline Weight & 0.05 & 0.08 & 0.07 & 0.18 \\
\hline
\end{tabular}

Table 9 The Relative Weight Values Among the Evaluation Indices of Supervision Aspect

\begin{tabular}{|c|c|c|c|c|}
\hline $\begin{array}{l}\text { Evaluation } \\
\text { index }\end{array}$ & $\begin{array}{l}\text { Enveronement } \\
\text { pohrution }\end{array}$ & 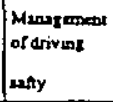 & $\begin{array}{l}\text { Manasment } \\
\text { orbu-itop } \\
\text { sowion }\end{array}$ & $\begin{array}{l}\text { Advertiscisknt } \\
\text { on bus }\end{array}$ \\
\hline Weight & 0.05 & 0.09 & 0.03 & 0.01 \\
\hline
\end{tabular}

Table 10 The Relative Weight Values Among the Evaluation Indices of Demand Aspect

\begin{tabular}{|c|c|c|}
\hline Evaluation & index & Weight \\
\hline \multirow{2}{*}{$\begin{array}{l}\text { Safty } \\
(0.2141)\end{array}$} & Accident rate & 0.15 \\
\hline & $\begin{array}{l}\text { Average vehicle } \\
\text { age }\end{array}$ & 0.07 \\
\hline \multirow{3}{*}{$\begin{array}{l}\text { Comfort } \\
(0.0727)\end{array}$} & $\begin{array}{l}\text { In-vehicle } \\
\text { congestion }\end{array}$ & 0.03 \\
\hline & $\begin{array}{l}\text { Proportion of air } \\
\text { condition } \\
\text { vehicle }\end{array}$ & 0.01 \\
\hline & Driving stable & 0.03 \\
\hline \multirow{2}{*}{$\begin{array}{c}\text { Convenience } \\
(0.0632)\end{array}$} & $\begin{array}{l}\text { Change bus } \\
\text { convenience }\end{array}$ & 0.02 \\
\hline & $\begin{array}{l}\text { Frequence } \\
\text { reliability }\end{array}$ & 0.04 \\
\hline Service & Attitude & 0.08 \\
\hline
\end{tabular}

within () is the weight

(2) The calculotion of each of the perfornance values of the evaluation indices.

The calculation is separated into two parts as quantified index and qualitative index. For quantified index, the values of its primary materials have to be traced back to the joint- managment center of bus companies, transportation department of Taipes municipal administration, traffic squad of the police department, environmental protection department: and each of the bus companies, while the information 
was garnered during the period of November 1992 to April 1993. Annong these items of information, since only insufficient information can be collected on the item "average vehicle age index", it was later replaced by another item "the ratio index of power weighted new vehicle" as in reference to the transportation department of Taipei City Administration (1993).

For qualitative index, the one as "bus-stop and station management index" has been continuously made used of as the transportation department owns information about it, while the performance values of the other five qualitative indices will be reckoned of their performance values by using the fuzzy ideas mentioned in the fourth part. This study has selected the largest transfer station -- the bus-stops in the periphery of Tajpei Railway Station -- to conduct random sample investigation of passenger, and valid questionnaire retrieved totals 250 copies. Atter which, the work of clarification procedures elucidated in the fourth part is proceeded.

There are five fuzzy expression levels being employed in the questionnaire of this study, which are very high, high, fair, low, very low and they are the ones in scale III (III of figure three). And the degree of membership of each of the fuzzy expressions after calculation are:u, (very

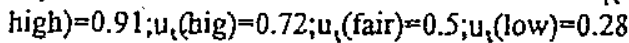

$: \mu$, (very low $)=0.09$, then these fuzzy sets can be rendered with such degree of membership to become the original performance value for qualitative indices.

Once the original performance value of each of the evaluation indices has been attained, the outcome will have to go through the process of vector standardization so as to achieve the consistency and comparability among each of the index units. Equations of vector standardization is as following: $r_{i j}=\frac{x_{y}}{\left[\bar{\Sigma} r_{i j}^{2}\right]^{\mu}}$

r: i operator of bus company

$j: j$ evaluation index

$1_{1}$. the performance value of evaluation index after vector standardization

$X_{1}$, the original performance value of the evaluation mdex

(3) Use TOPSIS method to conduct priority ranking and its results

This study has utilized TOPSIS method to conduct the final priority ranking because this method is easy to be understood, also the two basic premises of TOPSIS -- known attribute weight and its attribute being of monotonically increasing or monatonically decreasing - happen to be shared by the performance evaluation index of bus. Then, when the decision making matrix has been obtained after vector standardization seen in the above-mentioned procedure, the third step of TOPSIS -- to resolve for ideal solution and negative ideal solution -- can be rightly proceeded.

The basic notion of TOPSIS method is to consider the distance of each altemative (here refers to the operator of bus company) to the ideal solution and the negative ideal solution so that the selected alternative can be nearest to the ideal solution and farthest to the negative ideal solution. As a matter of course, the first thing is to locate the ideal solution $\left(V_{i}^{*}\right)$ and the negative ideal solution $\left(V_{i}^{*}\right)$ of each of the evaluation indices, then the distance of each bus company to the $\left(S_{i}{ }^{*}\right)$ ideal solution and the $\left(S_{1}\right)$ negative ideal solution is to be reckoned; at last, once the relative degree of nearness $\left(\mathrm{C}_{1}^{\circ}\right)$ of each bus company to the ideal solution is located, such value can then be based on to perform ranking. Of them.

$S_{i}^{+}=\left[\sum_{j=1}^{16}\left(V_{i j}-V_{j}^{+}\right)^{2}\right]^{12}$

$S_{i}=\left[\sum_{j=1}^{16}\left(V_{i j}-V_{j}^{-j}\right)^{2}\right]^{1 / 2}$

$C_{i}^{*}=\frac{s_{i}}{s_{i}+s_{i}}$

And the order of ranking of the ten bus companies obtained through TOPSIS method is:

Metropolitam $>$ Taipei $>$ HsinHsin $>$ SanChung $>$ TaNan $>$ (0.7481) (0.7355) (0.6801) (0.6695) (0.6390)

$\mathrm{T}_{a}$ Yo $>$ Municipal bus company $>$ Kwong. Hwa $>$

$\begin{array}{lll}(0.5707) \quad(0.5648) & (0.4727)\end{array}$

Chi Nan>Chung Hsin

(0.4719) $(0.3787)$

Within () is the degree of neamess revealing distance away from the ideal solution, and higher the numeral it tells shorter the distance. As can be learned from the result, the three bus companies as Metropolitan. Taipei, and Hsin Hsin perform the best and their degree of performance are respectively (100\% being the best) $78.41 \%, 73.55 \%$, and $68.01 \%$;. On the contrary, Chung Hsin, Chin Nan, and Kwong Hwa perform the worst, and their degree of performance are respectively $37.87 \%, 47.19 \%$, and $47.27 \%$.

And as can be informed from the annlysis results, Chung Hsin in accident rate (10). Chi $\mathrm{Nan}$ in costbenefit ratio (9), and Kwong Hwa in the management of driving safety $(10)$ perform the worst (( ) the number inside being the ranking), yet the weight values of these three indices rank in the foremost three, as a result, it tums out that the ranking of these three bus companies cannot move forward.

Should it be simply analyzed from the measurement results of the supply aspect, the four indices of the supply aspect would represent individually the most concerned parts to operators of 
bus companies as employee, vehicle, operation situation, and final eamings. Thus, the performance of management and operation of bus companies can be grasped and compared, and the order of ranking is as following:

It can be learned from the forgoing information that the management and operation performance of the municipal bus company does not only rank the last, its performance value is as well 0 in comparison to other nine bus companies. That would then be the spot of area which requires improvement, and it is as well the

primary reason why the integral ranking of the municipal bus company lists only the eighth.

If it is to be analyzed simply from the measurement result of the supply aspect, the eight indices of the demand aspect would then represent the degree of passenger satisfaction. Thus, the service quality of bus companies can be grasped and compared, and the order of the ranking is: From the preceding perspective of service quality, these ten bus companies can be grouped into four clusters: the municipal bus company performs the best, while Taipei, Metropolitan, San Chung and Hsin Hsin are of the next cluster, and Ta Nan, Chi Nan, Ta Yo rank the third, whereas Chung Hsin performs the worst.

\section{Vi. Conclusions}

For those past studies done on the performance evaluation of bus system, either their stress is focused on the measurement of demand aspect (the service standard to passenger) or on the measurement of supply aspect - (the management and operation performance of bus company operator), and the outcome obtained from either of the perspectives can hardly be fair and objective for both the passenger and bus company operator. So, this study would approach the issue from the viewpoint of controlled economy, divide the performance evaluation problem of bus system into three approach dominions supply, demand, and supervision, and establish respectively performance evaluation indices. Such practice can be more comprehensive and representative.

In view of the decision in regard to index weight, the relative weights among each of the hierarchies can be separately located through the concept of AHP hierarchical structure as well as the computation of eigenvector method, and this novel practice of resolution has become much more objective than any previous measure which considers all indices as consistent or locates its weight value through unrefined expert average point method. Furthermore, since consistency verification will be brought in during the calculation process in AHP, the derived weight would become more concise and reasonable.

On the administration of qualitative index, though more and more scholars have tried to bring in the idea of fuzziness, the fundamental spirit of fuzzy theory has oftentimes been ignored .- to reduce human subjective judgment. In this study, the focus is placed on clarifying those inherently fuzzy qualitative indices, while those quantifted indices with objective measurement values are handled separately, and double advantages is rendered by such employment as the problem becomes easier to handle and its nature is well accommodated. In addition, this study uses TOPSIS method to tank bus company operators because the fundamental principle of this procedure conforms to the prerequisite needed for ranking -- compatibility. And such particular feature of the method would furnish the ranking outcorie with more faimess.

At the end, two notions of ideas are put fonward in accordance to the results derived from the applied examples:

1. As can be learned from the ranking result of the supply aspect, the order of the municipal bus company ranks the last and its comparative performance is 0 , lagging behind other civilian bus companies a great deal of distance. Such a result tells that the management and operation of the municipal bus company is far from average and it also indicates the situation that, however strong the municipal bus company pushes for the privatisation policy, the reality seems to contrast its effor. Besides, there is not, at the meantime, enough legal enactments to protect the minority consumer groups and prevent the strike of bus company, therefore, efforts should be geared to improve the managment performance of bus company operators before a health system has been brought forh.

2. As can be learned from the ranking result of the demand aspect, the order of the municipal bus company ranks the first, and leads relatively a great deal ahead of other bus companies, such a result indicates the difference of salary system between civilian and municipal bus companies. Since the driver salary in civilian bus companies is usually calculated by bonus, phenomena as driver fighting for more passenger, speeding, and striving for more mileage are turned out that inevitably lowers service quality. Thus, how should the salary system of the municipal and civilian bus companies be improved is as well the other important task. 
Reference

1.Allen,W.G.and Frank DiCesare(1976), Transit Service Evaluation: Preliminary Identification of Variables Characterizing Level of Services. TRR 606, TRB, National Academy of Science, Washington, D.C., pp.41-47.

2.Alter,C.H., $\ulcorner$ Evaluation of Public Transit Service: The Level-of-Service Concept, (Abridgement),TRR 606,TRB, National Academy of Science, Washington,D.C.,pp.37-40.

3.Bakker,J.J.(1976), r Transit Operating Strategies and Level of Service J,TRR 606, TRB,National Academy of Science, Washington D.C.pp.I-5.

4.Chang,Y.U.andTasi,C.T.,(1993) ${ }^{\ulcorner}$Applications of fzzy theory to evaluation of bus transit system performance, ,Transportation Planning Journal,Vol.22,No.1,pp.70-104.

S.Chen S.J.and Hwang C.L.(1992), Fuzzy Multiple Attribute Decision Making,

Springer-Verlag, New York.

6. Fielding, G.J. and Anderson S.C. (1984), ${ }^{\top}$ PublicTransit Performance Evaluation: Application to Section 15 Data נ, Transportation Research Record 947, pp.1-7.

7.Fielding G.J.,Babitsky $T$. and Brenner M.E.(1985a), ' Performance Evaluation For Bus Transit J Transporation Research, Vol.19A, No.1,73-82.

8.Fielding,G.J.,Brenner,M.E.andKatherine

Faust(1985b). r Typology for Bus Transit 」 ,Transporation Research,Vol 19A,No.3,pp.269-278.

9.Fielding G.J.,Glauthier R.I. and Lave C.A.(1978), $\ulcorner$ Performance Indicater for Transit Management , Transportation, Vol.7, No.4, pp.365-379.

10.Hwang C.L. and Lin M.J. (1987), Group

Decision Making Under Multiple Criteria,Springer-Verlag,New York

11.Hwang C.L.and Lin M.J.(1981),Multiple Attribute Decision Making, Springer-Verlag,New York.

12.Lan,W.W.(1983), $r$ The study of level of service on different services zones in city, Transporation Planning Joumal, Vol.12, No.2, pp.127-192.

13.Saaty T.L.(1980), The Analytic Hierarchy Process, McGrawHill, New York.

14.Sugiyama K., Tagawa S. and Toda M. (1981),

$r$ Methods for visual Understanding of Hierarchial System Structures, IEEE Transationon Systems, Man and Cybernetics, SMC-11(2),pp.109-125.

15.Tally W.K. and Anderson P. (1981),

$r$ Effectiveness and Efficiency on Transit
Performance: A Theoritical Perspective, . Transportation Research, Vol.15A,No.6,pp.431-436. 16.Tanaboriboon Y., Quium A. and Changsingha C. (1993), r Performance Indicator Analysis: A Management Toll for The Improvement of Bus Transit Operation in Bangkok,, Journal of Advanced Transporation, Vol.27, No.2,pp.207-223. 17.Tranportation Department of Taipei Municipal Administration (1993), The operation and service indices evaluation repor in Taipei city during 1-6 1993.

18.Tzeng,G.H.andTeng,J.Y.(1983), $r$ The characteristicsand applicationsof AHP(-) , Journal of Chinese Statistic, Vol.27, No.6, pp.13707-13724.

19.Tzeng,G.H.andTeng,J.Y.(1983), ${ }^{r}$ The characteristics and applications of AHP (-) ) s Jounal of Chinese Statistic, Vol.27,No.7,pp.13767-13870. 